\title{
Elaborate mapping of the posterior visual pathway in awake craniotomy
}

\author{
${ }^{*}$ Tal Shahar, MD,1 Akiva Korn, MMedSc, DABNM,1 Gal Barkay, MD, ${ }^{1}$ Tali Biron, MSc, ${ }^{1}$ \\ Amir Hadanny, MD, ${ }^{1}$ Tomer Gazit, PhD, ${ }^{2}$ Erez Nossek, MD, ${ }^{1}$ Margaret Ekstein, MD, ${ }^{3}$ \\ Anat Kesler, MD, ${ }^{4}$ and Zvi Ram, MD ${ }^{1}$
}

\begin{abstract}
${ }^{1}$ Department of Neurosurgery, ${ }^{2}$ Tel-Aviv Center for Brain Functions, Wohl Institute for Advanced Imaging, ${ }^{3}$ Department of Anesthesiology and Intensive Care, and ${ }^{2}$ Department of Ophthalmology, Neuro-Ophthalmology Unit, Tel Aviv Medical Center (affiliated with Sackler Faculty of Medicine, Tel Aviv University), Tel Aviv, Israel
\end{abstract}

\begin{abstract}
OBJECTIVE Resection of intraaxial tumors adjacent to the optic radiation (OR) may be associated with postoperative visual field (VF) deficits. Intraoperative navigation using MRI-based tractography and electrophysiological monitoring of the visual pathways may allow maximal resection while preserving visual function. In this study, the authors evaluated the value of visual pathway mapping in a series of patients undergoing awake craniotomy for tumor resection.
\end{abstract}

METHODS A retrospective analysis of prospectively collected data was conducted in 18 patients who underwent an awake craniotomy for resection of intraaxial tumors involving or adjacent to the OR. Preoperative MRI-based tractography was used for intraoperative navigation, and intraoperative acquisition of 3D ultrasonography images was performed for real-time imaging and correction of brain shift. Goggles with light-emitting diodes were used as a standard visual stimulus. Direct cortical visual evoked potential (VEP) recording, subcortical recordings from the OR, and subcortical stimulation of the $\mathrm{OR}$ were used intraoperatively to assess visual function and proximity of the lesion to the OR. VFs were assessed pre- and postoperatively.

RESULTS Baseline cortical VEP recordings were available for 14 patients (77.7\%). No association was found between preoperative VF status and baseline presence of cortical VEPs $(p=0.27)$. Five of the 14 patients $(35.7 \%)$ who underwent subcortical stimulation of the OR reported seeing phosphenes in the corresponding contralateral VF. There was a positive correlation $(r=0.899, p=0.04)$ between the subcortical threshold stimulation intensity $(3-11.5 \mathrm{~mA})$ and the distance from the OR. Subcortical recordings from the OR demonstrated a typical VEP waveform in 10 of the 13 evaluated patients $(76.9 \%)$. These waveforms were present only when recordings were obtained within $10 \mathrm{~mm}$ of the $\mathrm{OR}(p=0.04)$. Seven patients (38.9\%) had postoperative VF deterioration, and it was associated with a length of $<8 \mathrm{~mm}$ between the tumor and the OR $(p=0.05)$.

CONCLUSIONS Intraoperative electrophysiological monitoring of the visual pathways is feasible but may be of limited value in preserving the functional integrity of the posterior visual pathways. Subcortical stimulation of the OR may identify the location of the OR when done in proximity to the pathways, but such proximity may be associated with increased risk of postoperative worsening of the VF deficit.

https://thejns.org/doi/abs/10.3171/2017.2.JNS162757

KEY WORDS visual evoked potential; awake craniotomy; brain tumor; optic radiation; electrophysiological monitoring; surgical technique

* Dr. Shahar and Mr. Korn contributed equally to this work. 
A CHIEVING maximal resection of intraaxial tumors is associated with improved outcome. Aggressive resection may, however, also result in new neurological deficits and deleteriously affect the patient's quality of life. Resection of tumors in the posterior temporal lobe or adjacent to the parietotemporooccipital junction may be associated with an injury to the optic radiation (OR), resulting in postoperative visual field (VF) deficits. Diffusion tensor imaging (DTI)-based tractography studies for subcortical white matter tracts combined with intraoperative navigation and electrophysiological studies are being used in an effort to avoid intraoperative white matter tract injury. Cortical and subcortical mapping has become the standard method to assess motor and language functions during resection of intraaxial tumors. However, the value of intraoperative mapping of the visual pathways during resection of tumors adjacent to the OR is unclear. In addition, the conclusions of recently published reports of the credibility and usefulness of visual pathway mapping are conflicting. ${ }^{2}$

Several reports have suggested that cortical visual evoked potentials (VEPs) can be reliably recorded intraoperatively and that they may predict postoperative visual function. ${ }^{11,15,18}$ Moreover, a report by Gras-Combe et al. has suggested that subcortical stimulation can reliably identify the OR in patients undergoing awake surgery for resection of low-grade gliomas involving the posterior visual pathways and thereby prevent visual dysfunction in most of them. ${ }^{8}$

We report our experience using multimodality electrophysiological techniques to map the posterior visual pathways during awake craniotomy in patients with intraaxial tumors. To our knowledge, this is the first time a combined array of electrophysiological studies has been used intraoperatively in the setting of awake tumor resection. Unlike recent studies that reported the authors' experience with cortical VEP recordings ${ }^{15}$ or subcortical stimulation of the $\mathrm{OR},{ }^{8}$ here we report our experience with the combined use of cortical VEP recordings and subcortical stimulation together with subcortical VEP recordings from the OR in an attempt to identify patients who may benefit from mapping the OR. To the best of our knowledge, the use of such subcortical VEP recordings in the setting of tumor resection has not been previously reported.

\section{Methods \\ Study Design}

This is a retrospective analysis of prospectively collected data from the brain tumor database of the Tel Aviv Medical Center. Approval from the institutional review board of Tel Aviv Medical Center was obtained. Data on neuro-ophthalmological, neuroimaging, and intraoperative electrophysiology studies in patients who underwent resection of tumors involving the posterior visual pathways, as well as intraoperative electrophysiology studies specific for the visual pathway, were retrieved from the medical records and evaluated. For intraoperative electrophysiological studies of the posterior visual pathway, we considered all patients older than 18 years who underwent awake craniotomy for resection of intraaxial tumors locat- ed within or adjacent to the OR. As a general guideline, we included patients who were able to cooperate and to tolerate the extended length of time under flash stimulation. The posterior visual pathways refer to the OR (also known as the geniculocalcarine tract), which is composed of axons arising in the lateral geniculate nucleus and terminating in the primary visual cortex of the calcarine fissure.

\section{Study Participants}

During the study period (May 2009 to March 2014), 18 patients (8 men and 10 women) with a mean age of $50.2 \pm$ 15.1 years (range 26-78 years) underwent awake craniotomy for tumor resection and intraoperative electrophysiological monitoring to evaluate the posterior visual pathway located within or adjacent to the OR. Patient characteristics, including tumor locations, pathological diagnosis, and preoperative VF evaluations, are listed in Table 1.

\section{Neuro-Ophthalmological Evaluation}

All patients underwent neuro-ophthalmological evaluations before and after surgery. Visual acuity was evaluated using the Snellen chart, and the VF was evaluated using the Humphrey automated perimetry. Mild homonymous hemianopia refers to cases in which only a partial (nondense) deficit was present.

\section{DTI-Based Tractography and Evaluation of the Tumor-to-OR Distance}

MRI was performed on a 3.0-T scanner (GE Sigma EXCITE HD). The protocol was that of conventional MRI (T1-weighted, T2-weighted, and FLAIR sequences); DTI; and contrast-enhanced T1-weighted sequences. DT images were acquired with $\mathrm{b}$ values of 0 and $1000 \mathrm{sec} /$ $\mathrm{mm}^{2}$ at 19 noncollinear gradient directions. Additional parameters included axial slices $3 \mathrm{~mm}$ in thickness, with no gap, covering the entire brain (FOV 200) and in-plane resolution of $0.7813 \times 0.7813 \mathrm{~mm}^{2}$. DTI analysis was performed using MRI Studio software (DTI Studio, version 2.4.01, October 2007). The DT (i.e., a $3 \times 3$ matrix) was calculated with a multivariate linear fitting algorithm for each voxel in the DTI analysis. The tensor in each voxel was spectrally decomposed to obtain its eigenvalues and eigenvectors, and the fiber direction at each voxel was assumed to be the eigenvector corresponding to the tensor's largest eigenvalue. This vector was color coded as follows: blue for superoinferior, red for left-right, and green for anteroposterior.

A streamline fiber tracking method was applied using DTI Studio software. A fiber assignment by continuous tracking algorithm and a brute-force reconstruction approach were used. For tracking of the OR, fiber tracking was initiated at a region of interest marked on coronal slices at the level of the occipital horn of the lateral ventricle (i.e., lateral to), including the so-called stratum sagittale. ${ }^{1}$ Fiber tracking was terminated when it reached a voxel with a fractional anisotropy $(\mathrm{FA})$ value of $<0.25$ or when the turning angle was $>70^{\circ}$. The fibers leading to the frontal lobe anterior to the lateral geniculate nucleus were then removed.

It should be noted that this procedure was used as a de- 
TABLE 1. Summary of patient characteristics

\begin{tabular}{|c|c|c|c|c|c|c|c|}
\hline Pt No. & Age (yrs) & Sex & Tumor Location & Laterality & Pathology & EOR $(\%)$ & Preop VF \\
\hline 1 & 57 & $\mathrm{~F}$ & Parietal & Rt & $\mathrm{AA}$ & NA & Mild It $\mathrm{HH}$ \\
\hline 2 & 58 & $M$ & Parietotemporal & $\mathrm{Lt}$ & $\mathrm{RCC}$ & 100 & Normal \\
\hline 3 & 78 & M & Parietal & $\mathrm{Lt}$ & GBM & 100 & Normal \\
\hline 4 & 26 & $\mathrm{~F}$ & Parietal & $\mathrm{Lt}$ & $\mathrm{AA}$ & 67.1 & Normal \\
\hline 5 & 69 & $M$ & Parietal & $\mathrm{Lt}$ & Melanoma & 100 & Normal \\
\hline 6 & 38 & $M$ & Parietotemporal & $\mathrm{Lt}$ & GBM & 53.6 & Rt HSQ \\
\hline 7 & 53 & M & Parietotemporal & $\mathrm{Lt}$ & GBM & NA & Mild rt HH \\
\hline 8 & 56 & $\mathrm{~F}$ & Temporal & $\mathrm{Lt}$ & AA & 100 & Normal \\
\hline 9 & 60 & $\mathrm{~F}$ & Temporooccipital & $\mathrm{Lt}$ & GBM & 100 & Rt HH \\
\hline 10 & 74 & $\mathrm{~F}$ & Parietal & $\mathrm{Lt}$ & GBM & 98.4 & Normal \\
\hline 11 & 37 & $\mathrm{~F}$ & Ant temporal & $\mathrm{Lt}$ & 0 & 73 & Normal \\
\hline 12 & 48 & $M$ & Ant temporal & $\mathrm{Lt}$ & $\mathrm{AA}$ & 42.8 & Normal \\
\hline 13 & 34 & M & Parietal & $\mathrm{Lt}$ & AA & 94.5 & Normal \\
\hline 14 & 59 & $\mathrm{~F}$ & Parietal & $\mathrm{Lt}$ & GBM & 100 & Mild rt HH \\
\hline 15 & 49 & M & Ant temporal & $\mathrm{Lt}$ & $\mathrm{AA}$ & 53.2 & Normal \\
\hline 16 & 39 & $\mathrm{~F}$ & Temporal & $\mathrm{Lt}$ & $\mathrm{AO}$ & 85.2 & Normal \\
\hline 17 & 39 & $\mathrm{~F}$ & Temporal & $\mathrm{Lt}$ & GBM & 100 & Normal \\
\hline 18 & 30 & $\mathrm{~F}$ & Parietal & $\mathrm{Rt}$ & 0 & 100 & Normal \\
\hline
\end{tabular}

$\mathrm{AA}=$ anaplastic astrocytoma; ant = anterior; $\mathrm{AO}=$ anaplastic oligodendroglioma; $\mathrm{GBM}=$ glioblastoma; $\mathrm{HH}=$ homonymous hemianopia; $\mathrm{HSQ}=$ homonymous superior quadrantanopia; $\mathrm{NA}=$ not available; $\mathrm{O}=$ oligodendroglioma; $\mathrm{Pt}=$ patient; $\mathrm{RCC}=$ renal cell carcinoma.

fault because the FA value was reduced in several specific cases in which reconstruction was incomplete. The decision to reduce the values of FA was based solely on anatomical considerations, and it was always performed prior to surgery, ruling out any possibility of bias due to feedback of intraoperative findings. Coregistration of the b0 signal intensity volume of the diffusion-weighted images with the spoiled gradient-recalled acquisition (SPGR) volume was conducted using SPM5 software (MathWorks). The generated fibers were saved as a binary mask for superimposing to the SPGR sequence after the coregistration procedure.

The new superimposed series were uploaded to the neuronavigation system. The iPlan 2.6 software (Brainlab) was used to measure the shortest distance between the reconstructed OR and the external surface of the tumor, as demonstrated by the T1-weighted signal with contrast material for enhancing tumors and by a T1-weighted hypointense signal abnormality for nonenhancing tumors. ${ }^{1}$

\section{Evaluation of Tumor Volume and Extent of Resection}

Volumetric assessment of the tumors was conducted using iPlan 3.0 cranial software (Brainlab). The area corresponding to the tumor was manually segmented across all MRI sections. In cases of tumors showing contrast enhancement, only the volume bound by contrast enhancement was segmented. For tumors not showing contrast enhancement, the entire area of T1-weighted hypointense signal abnormality was segmented. Each patient was evaluated with preoperative and immediate postoperative MRI. In 2 cases, the extent of resection (EOR) could not be evaluated because of missing postoperative MRI studies.

\section{Awake Craniotomy and Operative Technique}

An extended description of the awake craniotomy procedure has been previously published. ${ }^{14}$ In brief, patients received small doses of intravenous sedative and analgesic medications on arrival to the operating room. Scalp nerve blocks were done according to the location of the planned pinning and incision site immediately before the pinning procedure in all patients. Scalp subdermal needle electrodes were placed in the locations described below. Monitoring of anesthesia was standard and additionally included invasive blood pressure monitoring. Spontaneous ventilation was monitored by capnography. Urinary catheters were usually not inserted to reduce the awake patient's discomfort, and mannitol $(0.5-1 \mathrm{~g} / \mathrm{kg})$ was only used in selected cases of significantly increased intracranial pressure.

Light sedation was achieved intraoperatively with continuous administration of remifentanil, and the entire procedure was well tolerated by the majority of patients. When sedation with remifentanil was not sufficient, careful supplementation with propofol was used. All sedatives or analgesics were discontinued briefly after head fixation and before the beginning of skin incision to allow patient cooperation. Patients who experienced pain from dural manipulation received lidocaine $1 \%$ infiltrated between the dural leaves along branches of the meningeal arteries. Mild sedation and pain control medications were given after the resection was completed until closure of the skin incision.

\section{Neuronavigation and Intraoperative Imaging}

The SonoWand navigation system and the Brainlab navigation system were used for intraoperative neuronavi- 
gation. Prior to the surgery, preoperative MRI anatomical studies (fast SPGR), including the tractography data of the OR, were uploaded to the system. Baseline ultrasonography (US) was done after the craniotomy, and the US scan was integrated into the navigation database. Multiple acquisitions of US scans were made intraoperatively to evaluate and compensate for brain shift. A scaled 10$\mathrm{mm}$ virtual extension to the wand tip was displayed on the navigation screen during navigation assessment for calculation of the distance of the navigation probe's point to the OR fibers as depicted by DTI. This $10-\mathrm{mm}$ offset was used as a scale marker for offline quantification of the distance between the probe location and the DTI-depicted OR tracts on screenshot images.

\section{Cortical and Subcortical VEP Recordings}

VEPs were collected following biocular flash stimulation using light-emitting diodes embedded in goggles (Medtronic NIM Eclipse system). Pulse widths were 15 microseconds and administered at a rate of 1.2 stimulations per second. Recordings were made from the subdural space over the occipital cortex using the distal 3 contacts of an 8-contact subdural strip electrode (PMT Corporation) with 1-cm spacing between contacts. The electrode was placed over the occipital lobe, and 500-msec, timelocked signal sweeps were recorded and averaged between 10 and 50 times until an acceptable signal-to-noise ratio was obtained.

A bandwidth of 1-50 Hz was used for cortical recordings. For subcortical recordings, a bayonet-style monopolar probe was used (inomed) and held at the depth of the tumor cavity at regions of interest determined by the surgeon. The bandwidth for subcortical recordings was established as $1-300 \mathrm{~Hz}$ to allow for recording of faster activity inherent to potentials traveling across white matter. A subdermal needle electrode placed at the FPz landmark on the upper forehead was used as a signal reference for both methods. Signals $>100 \mu \mathrm{V}$ were automatically rejected from the average in both methods. Cortical recordings were always performed concurrently during attempts to record subcortical activity.

Comparisons with scalp-based VEP normative data were not made; rather, each patient served as his or her own control. VEP recordings, whether cortical or subcortical, were established and verified as positive when at least 2 reproducible waveforms were evident, with similar morphological characteristics and latency and amplitude values. Analysis of both the cortical and subcortical waveforms consisted of measurement of the amplitude of dominant positive and negative peaks within the waveform, as well as the onset latency of the first negative or positive deflection. A typical VEP is defined as a waveform containing a negative peak at approximately $75 \mathrm{msec}$ and a positive peak at approximately $100 \mathrm{msec} \cdot{ }^{19}$ An early latency response was defined when the onset latency of the subcortically recorded VEP waveform preceded $65.0 \mathrm{msec}$.

\section{Subcortical Stimulation of the OR}

Subcortical stimulation was undertaken using a bayonet-style monopolar probe (inomed) connected to the intraoperative evoked potential unit. Two-second trains of $50-\mathrm{Hz}$ stimulation (biphasic, 500-microsecond pulse width) were delivered at stepwise increasing intensities ranging from 2 to $15 \mathrm{~mA}$, with at least a 5-second pause between the 2-second trials. The return subdermal needle electrode was placed in the subcutaneous tissue in the anterior aspect of the surgical wound at the farthest distance from the occipital area to avoid stimulation of the occipital cortical-based visual system, which would contribute to a false-positive report. Electrocorticographic recordings were undertaken during all stages of electrical stimulation to detect epileptic afterdischarges that could contribute to false-positive mapping or clinical seizures.

\section{Statistical Analysis}

Data are expressed as the mean \pm SD for parametric variables and frequencies and as percentages for nonparametric variables. A univariate analysis was performed using Fisher's exact test to identify significant variables $(p<0.05)$. Normality of parametric data was performed using the Kolmogorov-Smirnov (KS) test. A 2-tailed Student t-test for independent samples was used to compare parametric variables according to KS test results. Pearson's/Spearman's correlations were performed between parametric variables according to KS test results. All data were analyzed using a commercial statistical package (SPSS version 21 software).

\section{Results}

\section{Cortical VEP Recording}

Eighteen patients underwent awake craniotomy for the resection of intraaxial tumors with electrophysiological monitoring of the posterior visual pathway (Table 1). Baseline cortical VEP recordings were available for 14 patients (77.7\%). The lack of these recordings in the other 4 patients stemmed in 1 case (Patient 5) from the patient's discomfort with the placement of flash goggles and from the absence of an isolated, synchronized, and reproducible cortical VEP in the remaining 3 cases. There was no association between the preoperative VF status and the baseline presence of cortical VEPs recorded prior to tumor resection ( $\mathrm{p}=0.27$; Fig. 1). Only 9 of the 13 patients (69.2\%) with a normal preoperative VF had baseline VEPs within normal limits. All 5 patients with preoperative VF deficits demonstrated baseline cortical VEPs within normal limits (Table 2 describes intraoperative electrophysiology studies).

\section{Subcortical Stimulation of the OR}

Subcortical stimulation was not applicable to all patients due to either physical discomfort or the presence of afterdischarge potentials to prior cortical stimulation for motor or language mapping. Patients who were considered highly susceptible to epileptic seizures, as demonstrated by prior afterdischarge potentials, were excluded from subcortical mapping. Five of the 14 patients (35.7\%) who underwent subcortical stimulation of the OR experienced phosphenes in the contralateral VF. Four of these 5 patients had an intact preoperative $\mathrm{VF}$, and the fifth had a homonymous quadrantanopia on the preoperative VF assessment. 


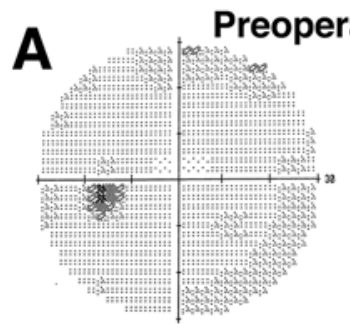

Left eye

\section{Cortical VEP Baseline}

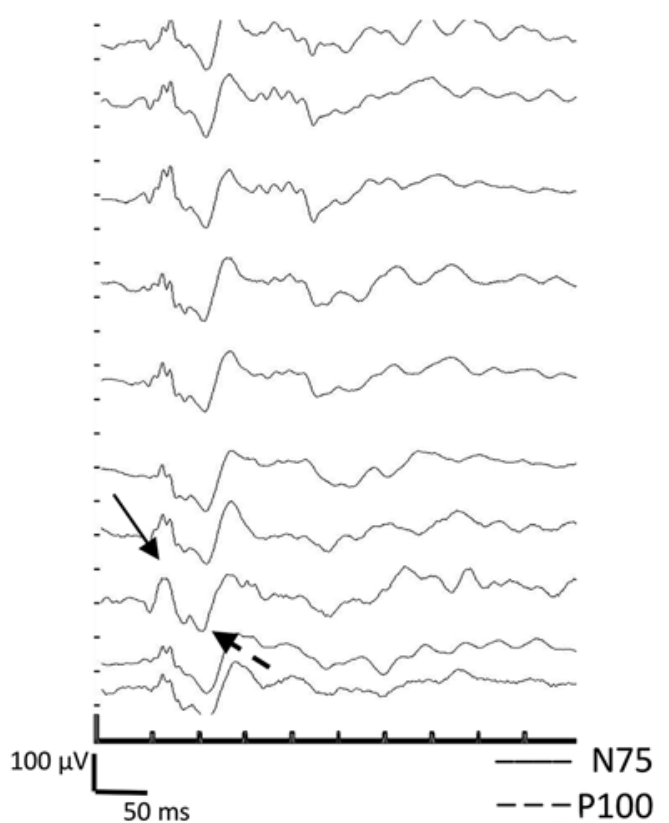

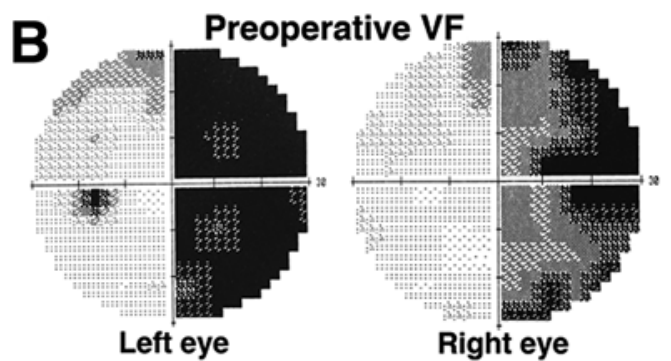

Cortical VEP Baseline

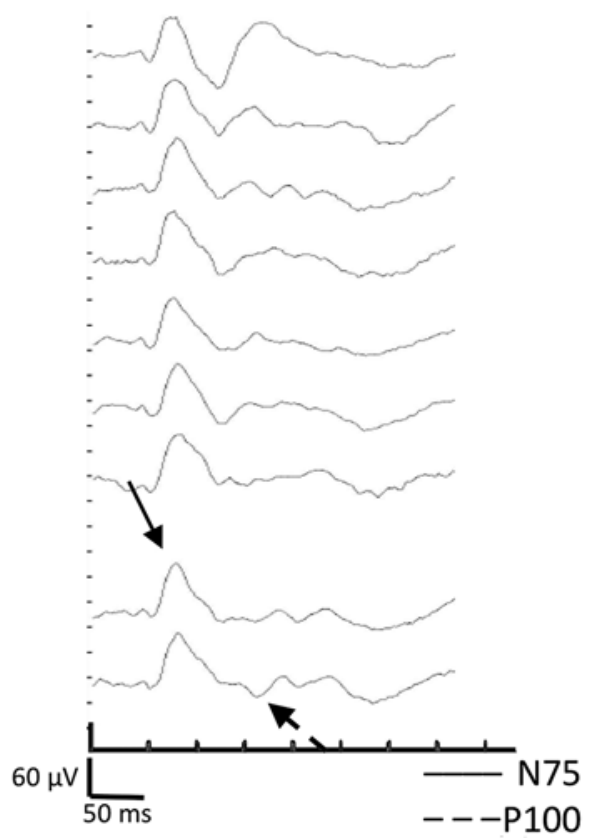

FIG. 1. Baseline cortical VEP recording made using subdural electrodes positioned at the occipital pole in a patient with an intact preoperative VF $(\mathbf{A})$ and in a patient with a significant VF deficit (B). Note the similar VEP appearance regardless of VF status.

The point of subcortical stimulation in these 5 patients was $<10 \mathrm{~mm}$ from the OR, as depicted on the intraoperative navigation system and based on the reconstructed MR-DT images. Moreover, there was a significant correlation $(r=0.899, p=0.04)$ between the intensity of the subcortical threshold stimulation $(3-11.5 \mathrm{~mA}$ ) and the distance from the OR, as depicted by the reconstructed MR-DT images. Interestingly, subcortical stimulation of the OR did not elicit a visual response in the 2 cases where the tumor was located within the OR. These 2 patients were also characterized as having preoperative mild-todense homonymous hemianopia (Table 2).

\section{Subcortical Recordings From the OR}

Subcortical recordings from the OR were attempted in 13 patients $(72.2 \%)$, and the results were positive in 10 of them (76.9\%). In 5 patients, subcortical VEP recordings were not attempted due to the patient's discomfort. Subcortical recordings from a location $\leq 10 \mathrm{~mm}$ from the OR (as depicted on the intraoperative navigation system) were associated with a positive appearance of the VEP waveform $(\mathrm{p}=0.04$; Fig. 2). Successful subcortical recording from the OR was not associated with the preoperative VF status $(\mathrm{p}=0.5)$ nor with reporting of experiencing phosphenes in response to subcortical stimulation $(\mathrm{p}=0.46)$. However, successful subcortical recordings were evident in all 5 patients who reported that they did experience phosphenes upon subcortical stimulation. Subcortical VEP recordings of the OR were successful in both cases in which the tumor was located within the OR (Table 2).

\section{Postoperative VF Deterioration}

Postoperative deterioration of the VF occurred in 7 patients (38.9\%). The experience of phosphenes following subcortical stimulation was associated with postoperative VF deterioration, suggesting involvement of the OR at the site of stimulation. Four of the 5 patients who reported experiencing phosphenes following subcortical stimulation had worsened VFs postoperatively. Abnormal preoperative VFs or successful cortical or subcortical VEP recordings were not associated with worsened postoperative visual function. The minimal distance of a tumor to the OR of $<8 \mathrm{~mm}$ was associated with a worse outcome ( $\mathrm{p}=$ 0.05 ). We found no association between EOR and the out- 
TABLE 2. Summary of patient characteristics including intraoperative electrophysiology studies

\begin{tabular}{|c|c|c|c|c|c|c|c|c|}
\hline $\begin{array}{l}\text { Pt } \\
\text { No. }\end{array}$ & $\begin{array}{c}\text { Tumor-to-OR } \\
\text { Distance }(\mathrm{mm})\end{array}$ & $\begin{array}{c}\text { Baseline } \\
\text { Cortical VEP }\end{array}$ & $\begin{array}{l}\text { Attempted Subcortical } \\
\text { Stimulation of OR }\end{array}$ & $\begin{array}{c}\text { Response to } \\
\text { Subcortical Stimulation }\end{array}$ & $\begin{array}{c}\text { Attempted Subcortical } \\
\text { Recording }\end{array}$ & $\begin{array}{l}\text { Subcortical } \\
\text { Recording }\end{array}$ & $\begin{array}{l}\text { Postop } \\
\text { VF }\end{array}$ & $\begin{array}{l}\text { Postop VF } \\
\text { Outcome }\end{array}$ \\
\hline 1 & 9.3 & Present & No & NA & Yes & Present & Mild It HH & Stable \\
\hline 2 & 38.5 & Present & No & NA & Yes & Absent & Normal & Stable \\
\hline 3 & 2 & Present & Yes & $3 \mathrm{~mA}$ & Yes & Present & Mild rt HH & Worse \\
\hline 4 & 2 & Present & Yes & $3 \mathrm{~mA}$ & Yes & Present & Rt HIQ & Worse \\
\hline 5 & 17.5 & NA & Yes & Absent & No & NA & Normal & Stable \\
\hline 6 & 7 & Present & Yes & $11.5 \mathrm{~mA}$ & Yes & Present & Rt HH & Worse \\
\hline 7 & w/in & Present & Yes & Absent & Yes & Present & Rt HH & Worse \\
\hline 8 & 1 & Absent & Yes & Absent & Yes & Absent & Normal & Stable \\
\hline 9 & w/in & Present & Yes & Absent & Yes & Present & Rt HH & Stable \\
\hline 10 & 1 & Present & Yes & Absent & Yes & Present & Normal & Stable \\
\hline 11 & 1 & Present & Yes & Absent & Yes & Present & Rt HIQ & Worse \\
\hline 12 & 1 & Absent & No & NA & No & NA & Normal & Stable \\
\hline 13 & 19.7 & Present & Yes & Absent & No & NA & Normal & Stable \\
\hline 14 & 1 & Present & Yes & Absent & No & NA & Rt HH & Worse \\
\hline 15 & 1 & Absent & No & NA & No & NA & Normal & Stable \\
\hline 16 & 2 & Present & Yes & $5 \mathrm{~mA}$ & Yes & Present & Normal & Stable \\
\hline 17 & 10.5 & Present & Yes & Absent & Yes & Absent & Normal & Stable \\
\hline 18 & 7 & Present & Yes & $8 \mathrm{~mA}$ & Yes & Present & $\mathrm{Lt} \mathrm{HH}$ & Worse \\
\hline
\end{tabular}

$\mathrm{HIQ}=$ homonymous inferior quadrantanopia; NA = not available due to nonattempt; $w /$ in = the tumor was found within the OR.

come of VF in our cohort (OR 0.99; 95\% CI 0.94-1.04; $\mathrm{p}$ $=0.62)$. There was no significant difference $(p=0.64)$ between the mean EOR of patients characterized with stable VF $(87.4 \% \pm 21.4 \%)$ and that of patients with worse VF $(82.3 \% \pm 20.4 \%)$ postoperatively.

\section{Tumor Pathology and Intraoperative Electrophysiological Findings}

To examine a possible association between tumor pathology and our electrophysiological findings, we divided our cohort into 3 subgroups of pathology that included high-grade glioma $(n=14)$, low-grade glioma $(n=2)$, and metastasis $(n=2)$. We did not find an association between tumor pathology and the presence of cortical VEPs at baseline $(\mathrm{p}=0.68)$, subcortical recording $(\mathrm{p}=0.14)$, or response to subcortical stimulation $(\mathrm{p}=0.69)$. No association was found between the glioma grades and the presence of cortical VEPs at baseline $(\mathrm{p}=0.74)$, subcortical recording $(p=0.09)$, or response to subcortical stimulation $(p=0.73)$. These findings should be considered with caution because of the small sample size.

\section{Discussion}

Intraoperative electrophysiological monitoring of motor function is commonly used during resection of tumors near the corticospinal tract. However, the role and value of electrophysiological monitoring of posterior visual pathways have yet to be determined. This study describes the use of elaborate intraoperative electrophysiological monitoring of the OR during awake resection of intraaxial tumors. It emerged that successful baseline cortical VEP recordings were not associated with the preoperative VF status. Only patients with an intact or near-intact preoperative VF reported experiencing phosphenes following subcortical stimulation of the OR.

Moreover, the intensity of the threshold stimulation correlated positively with the distance from the OR, as long as the distance from the OR was $<10 \mathrm{~mm}$. Similarly, subcortical recording could only be obtained when performed at a distance $<10 \mathrm{~mm}$ from the OR. Interestingly, the report of experiencing phosphenes following subcortical stimulation of the OR was associated with a worsening postoperative VF. This might be explained by the relatively tenuous nature of the OR and complexity of the visual system, leading to postoperative deficits following surgical manipulation within or adjacent to the OR. This is in contrast to surgery adjacent to the corticospinal tract, where motor deficits can be avoided even when surgical manipulation is performed within 3-6 $\mathrm{mm}$ of the motor pathways..$^{13,16}$

The value of intraoperative monitoring of cortically recorded VEPs has been a matter of debate for the last 4 decades. ${ }^{5}$ Early reports suggested that cortical VEP monitoring may be used intraoperatively to monitor the functional status of the visual system. Those reports also suggested that such monitoring may help prevent damage during the resection of lesions that compress the anterior visual pathway (such as sellar and suprasellar lesions), tumors associated with posterior visual pathways, as well as in basal ganglia lesionectomies. It was also suggested that cortical VEP monitoring may be able to predict visual outcome. . $^{3,5,9,15,20}$

More recent reports have highlighted the limitations and questioned the reliability of intraoperative VEP monitoring, suggesting that there was no correlation between 

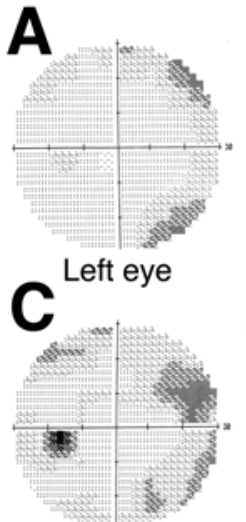

Left eye

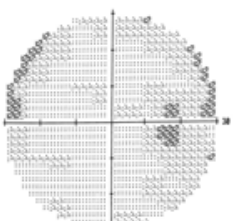

Right eye

\section{$\mathbf{B}$}

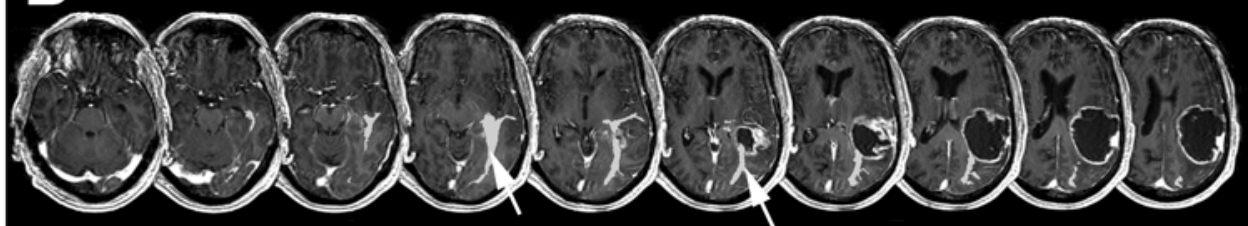

D

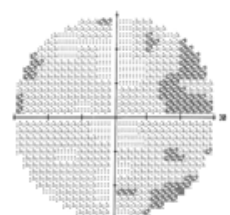

Right eye
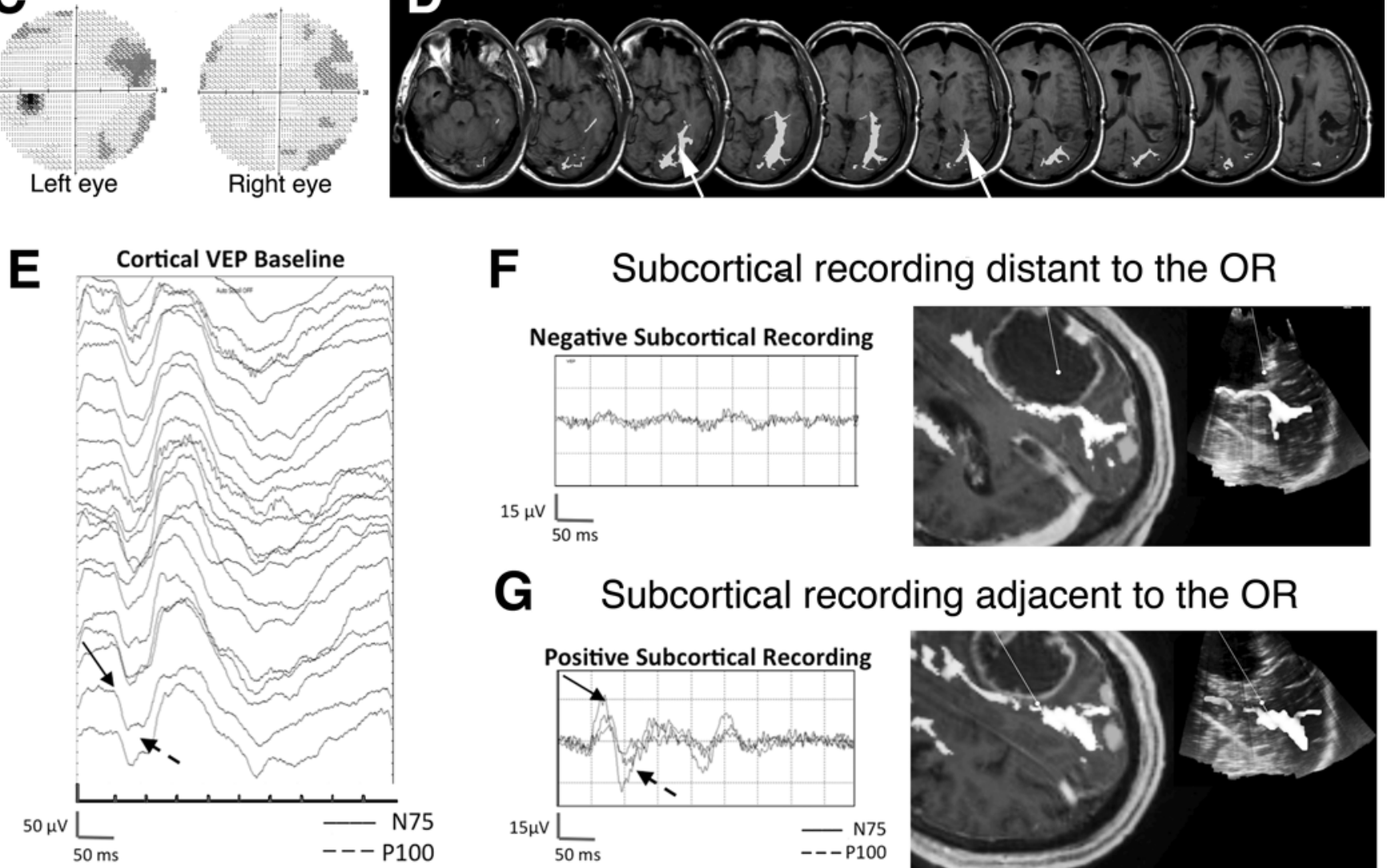

G Subcortical recording adjacent to the OR
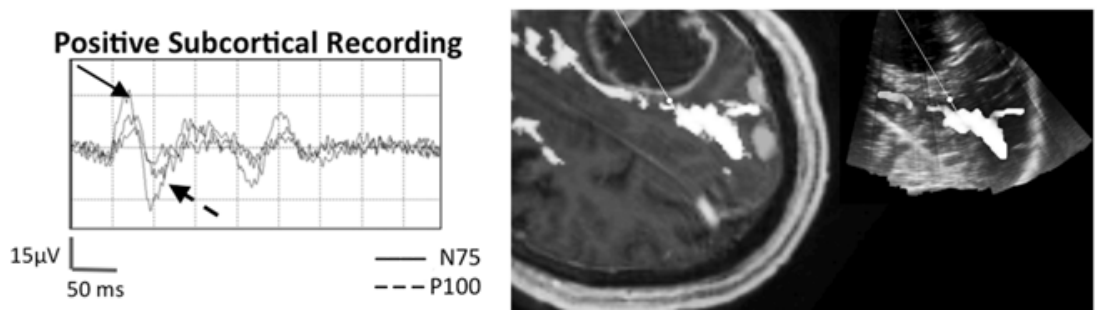

FIG. 2. Patient 3. This 78-year-old man underwent awake craniotomy for resection of a newly diagnosed left parietal glioblastoma. A VF test at baseline (A) demonstrated a very mild upper-right quadrantanopia. Preoperative axial contrast-enhanced T1-weighted MR image of a left parietal ring-enhancing lesion (B) demonstrating proximity $(<5 \mathrm{~mm})$ of the tumor to the reconstructed DT images of the OR (white arrows). Cortical VEP recording prior to tumor resection (E) using a subdural electrode positioned toward the occipital pole exhibited a typical N75 appearance (solid arrow) and a P100 (dashed arrow) component of a VEP waveform. A subcortical recording distant from the OR failed to demonstrate the typical VEP appearance (F, left panel). The location of the recording probe (F, right panel) was demonstrated intraoperatively by the navigation system using the preoperative MR image ( $F$, right panel, left side) and intraoperative US-based navigation ( $F$, right panel, right side) to account for brain shift. Subcortical recording $2 \mathrm{~mm}$ from the OR, as depicted by the navigation system using the preoperative MR image (G, right panel, left side) and intraoperative US-based navigation (G, right panel, right side), demonstrated a typical VEP waveform (G, left panel). Note the proximity of the recording probe in the superimposed reconstructed OR to the US image in an offline analysis (G, right panel, right side). Postoperative MR image (D) demonstrated complete resection of visible tumor and integrity of the reconstructed OR (white arrows). The postoperative VF test results suggest a mild worsening deficit, predominantly in the upper-right VF (C).

intraoperative VEP changes and VF outcome.,17 Ota et al. considered that low signal intensity and poor spatial resolution of VEPs might be explained by the use of volatile anesthesia and the use of recording from scalp VEPs rather than directly from the brain surface. ${ }^{15}$ They and others suggested that direct VEP recording using subdural electrodes under general anesthesia allows reliable monitoring of the functional integrity of the posterior visual pathway. ${ }^{10,15}$
In the current study in awake patients, the use of subdural electrodes placed close to the primary visual cortex showed no association between either preoperative VF status or postoperative outcome of visual function and intraoperative recordings of cortical VEPs, similar to the aforementioned papers.

Indeed, the presence of a robust cortical VEP at baseline in visually compromised patients is of concern and brings into question the value of this technique. One possible ex- 
planation of this phenomenon is that the VEPs generated by the intact contralateral visual cortex can mask the presumed VEP waveform deficiencies generated by the ipsilateral visual system. It is also possible that the patient's VF deficits were the result of a type of so-called cortical blindness with a source downstream to the primary visual system, despite intact conduction of the relevant OR. ${ }^{6,7} \mathrm{Ei}-$ ther of these possibilities would support a recordable and monitorable waveform baseline despite a significant clinical visual deficit, and therefore do not provide or advance a practical solution to the problem of detecting iatrogenic injury to the $\mathrm{OR}$ at risk.

Awake intraoperative mapping of the OR by subcortical stimulation was first reported by Duffau et al. in $2004 .{ }^{4}$ In their extended description of the technique, those authors proposed that direct subcortical stimulation of the OR is a reliable and effective method to preserve visual function. ${ }^{8}$ All 14 patients included in that study had an intact preoperative VF test result, and most $(85.7 \%)$ were diagnosed as having low-grade oligodendroglia involving the OR. They all reported visual symptoms during stimulation with an intensity of 2-4 mA, including blurred vision (78.6\%), phosphenes (28.6\%), and impression of shadows (14.3\%).

In the current study, mapping on the basis of phosphenes was successful in $35.7 \%$ of attempts and, with the exception of 1 patient with a mild preoperative VF deficit, it was successful in all patients who had an intact VF. This is compared with the VEP recording-based OR mapping, where the success rate was $66 \%$ of all attempts and it was not associated with preoperative VF integrity status. It would be reasonable to hypothesize that even a disrupted visual pathway can support a subcortical VEP recording as long as the anterior portion of the pathway is intact. Thus, a recording made at the anterior deep aspect of the tumor cavity, where OR fibers are activated in response to the visual stimulus, can still support VEP production.

The presence of phosphenes, on the other hand, may be more dependent upon cortical processing of an activated white matter system and therefore may depend on a more intact visual system. Moreover, a short distance $(<10 \mathrm{~mm})$ from the point of the subcortical stimulation to the OR is apparently required for it to elicit phosphenes. In those cases, the stimulation intensity $(3-11.5 \mathrm{~mA})$ was correlated with the distance from the OR, but it was not in cases in which the tumor had invaded the OR. The latter patients did not report experiencing phosphenes upon stimulation of the OR, probably due to disruption of the OR integrity by the tumor.

\section{Conclusions}

Intraoperative electrophysiological monitoring of the visual pathways is feasible. However, it could be postulated that both phosphene-evoked stimulation and VEP recordings are limited in their utility as warning tools to alert the surgeon to an imminent anatomical disruption of the OR. It is possible that triggering of phosphene production by stimulation is feasible only when the stimulation site is too close to the OR, thus leading to a visual deficit. Intriguingly, when we looked at a possible association be- tween EOR and postoperative visual deficit, we could not demonstrate a causal relationship. These findings should be considered with caution because of the small sample size. In addition, the subcortical recording could elicit a false-positive response if the recording site is anterograde to the damaged site. However, as in many other contexts of neurophysiological-based mapping, positive mapping does not imply intact functional integrity, ${ }^{12}$ and it should not be assumed.

\section{References}

1. Catani M, Jones DK, Donato R, Ffytche DH: Occipito-temporal connections in the human brain. Brain 126:2093-2107, 2003

2. Cedzich C, Schramm J, Fahlbusch R: Are flash-evoked visual potentials useful for intraoperative monitoring of visual pathway function? Neurosurgery 21:709-715, 1987

3. Costa e Silva I, Wang AD, Symon L: The application of flash visual evoked potentials during operations on the anterior visual pathways. Neurol Res 7:11-16, 1985

4. Duffau H, Velut S, Mitchell MC, Gatignol P, Capelle L: Intra-operative mapping of the subcortical visual pathways using direct electrical stimulations. Acta Neurochir (Wien) 146:265-270, 2004

5. Feinsod M, Selhorst JB, Hoyt WF, Wilson CB: Monitoring optic nerve function during craniotomy. J Neurosurg 44:2931, 1976

6. Frank Y, Torres F: Visual evoked potentials in the evaluation of "cortical blindness" in children. Ann Neurol 6:126-129, 1979

7. Good WV, Jan JE, Burden SK, Skoczenski A, Candy R: Recent advances in cortical visual impairment. Dev Med Child Neurol 43:56-60, 2001

8. Gras-Combe G, Moritz-Gasser S, Herbet G, Duffau H: Intraoperative subcortical electrical mapping of optic radiations in awake surgery for glioma involving visual pathways. J Neurosurg 117:466-473, 2012

9. Handel N, Law J, Hoehn R, Kirsch W: Monitoring visual evoked response during craniofacial surgery. Ann Plast Surg 2:257-258, 1979

10. Kamada K, Todo T, Morita A, Masutani Y, Aoki S, Ino K, et al: Functional monitoring for visual pathway using real-time visual evoked potentials and optic-radiation tractography. Neurosurgery 57 (1 Suppl):121-127, 2005

11. Kodama K, Goto T, Sato A, Sakai K, Tanaka Y, Hongo K: Standard and limitation of intraoperative monitoring of the visual evoked potential. Acta Neurochir (Wien) 152:643648,2010

12. Morota N, Ihara S, Deletis V: Intraoperative neurophysiology for surgery in and around the brainstem: role of brainstem mapping and corticobulbar tract motor-evoked potential monitoring. Childs Nerv Syst 26:513-521, 2010

13. Nossek E, Korn A, Shahar T, Kanner AA, Yaffe H, Marcovici $\mathrm{D}$, et al: Intraoperative mapping and monitoring of the corticospinal tracts with neurophysiological assessment and 3-dimensional ultrasonography-based navigation. Clinical article. J Neurosurg 114:738-746, 2011

14. Nossek E, Matot I, Shahar T, Barzilai O, Rapoport Y, Gonen $\mathrm{T}$, et al: Intraoperative seizures during awake craniotomy: incidence and consequences: analysis of 477 patients. Neurosurgery 73:135-140, 2013

15. Ota T, Kawai K, Kamada K, Kin T, Saito N: Intraoperative monitoring of cortically recorded visual response for posterior visual pathway. J Neurosurg 112:285-294, 2010

16. Prabhu SS, Gasco J, Tummala S, Weinberg JS, Rao G: Intraoperative magnetic resonance imaging-guided tractography with integrated monopolar subcortical functional mapping 
for resection of brain tumors. Clinical article. J Neurosurg 114:719-726, 2011

17. Raudzens PA: Intraoperative monitoring of evoked potentials. Ann N Y Acad Sci 388:308-326, 1982

18. Sasaki T, Itakura T, Suzuki K, Kasuya H, Munakata R, Muramatsu $\mathrm{H}$, et al: Intraoperative monitoring of visual evoked potential: introduction of a clinically useful method. J Neurosurg 112:273-284, 2010

19. Tandon PN, Ramamurthi R: Ramamurthi and Tandon's Textbook of Neurosurgery, ed 3. New Delhi: Jaypee, 2012

20. Tobimatsu S, Shima F, Ishido K, Kato M: Visual evoked potentials in the vicinity of the optic tract during stereotactic pallidotomy. Electroencephalogr Clin Neurophysiol 104:274-279, 1997

\section{Disclosures}

The authors report no conflict of interest concerning the materi- als or methods used in this study or the findings specified in this paper.

\section{Author Contributions}

Conception and design: Ram, Shahar, Korn. Acquisition of data: Ram, Shahar, Korn, Barkay, Biron, Gazit, Nossek, Kesler. Analysis and interpretation of data: Ram, Shahar, Korn, Biron, Hadanny, Nossek, Kesler. Drafting the article: Shahar, Korn, Barkay, Biron, Hadanny, Gazit, Nossek. Critically revising the article: all authors. Reviewed submitted version of manuscript: all authors. Approved the final version of the manuscript on behalf of all authors: Ram. Statistical analysis: Hadanny. Administrative/ technical/material support: Barkay, Gazit, Ekstein. Study supervision: Ram.

\section{Correspondence}

Zvi Ram, Department of Neurosurgery, Tel Aviv Medical Center, 6 Weizmann St., Tel Aviv 6423906, Israel. email: zviram@tlvmc. gov.il. 